\title{
Right-hemispheric cortical contributions to language ability in healthy adults
}

\author{
Helene van Ettinger-Veenstra, Mattias Ragnehed, Anita McAllister, \\ Peter Lundberg and Maria Engström
}

\section{Linköping University Post Print}

N.B.: When citing this work, cite the original article.

Original Publication:

Helene van Ettinger-Veenstra, Mattias Ragnehed, Anita McAllister, Peter Lundberg and Maria Engström, Right-hemispheric cortical contributions to language ability in healthy adults, 2012, Brain and Language, (120), 3, 395-400.

http://dx.doi.org/10.1016/j.band1.2011.10.002

Copyright: Elsevier http://www.elsevier.com/

Postprint available at: Linköping University Electronic Press

http://urn.kb.se/resolve?urn=urn:nbn:se:liu:diva-73623 


\section{Right-Hemispheric Cortical \\ Contributions to Language Ability in Healthy Adults.}

\section{Running title: Language Ability in Adults}

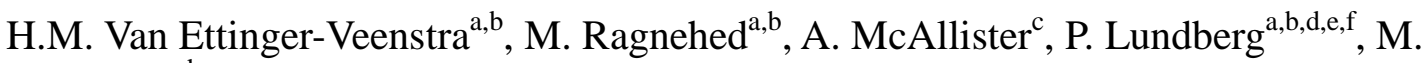
Engström ${ }^{\mathrm{a}, \mathrm{b}}$
a. Center for Medical Image Science and Visualization (CMIV), Linköping University, Linköping, Sweden
b. Department of Medical and Health Science, Division of Radiology, Linköping University, Linköping, Sweden
c. Department of Clinical and Experimental Medicine, Division of Logopedics, Linköping University, Linköping, Sweden
d. Department of Medical and Health Science, Division of Radiation Physics, Linköping University, Linköping, Sweden
e. Department of Radiation Physics, CKOC, University Hospital of Linkoping, Sweden.
f. Department of Radiology, CKOC, University Hospital of Linkoping, Sweden.

\section{All correspondence should be sent to:}

Helene Van Ettinger - Veenstra, MSc

Center for Medical Image Science and Visualization (CMIV)

Linköping University/US

SE-581 85 Linköping, Sweden

Tel: +46 (0)101038986

Email: helene.veenstra@liu.se

21 pages, 1 table and 2 figures 


\section{LANGUAGE ABILITY IN ADULTS}

\section{Abstract}

In this study we investigated the correlation between individual linguistic ability based on performance levels and their engagement of typical and atypical language areas in the brain. Eighteen healthy subjects between 21 to 64 years participated in language ability tests, and subsequent functional MRI scans measuring brain activity in response to a sentence completion and a word fluency task. Performance in both reading and high-level language tests correlated positively with increased right-hemispheric activation in the inferior frontal gyrus (specifically Brodmann area 47), the dorsolateral prefrontal cortex (DLPFC), and the medial temporal gyrus (Brodmann area 21). In contrast, we found a negative correlation between performance and left-hemispheric DLPFC activation.

Our findings indicate that the right lateral frontal and right temporal regions positively modulate aspects of language ability.

Keywords: Linguistics; Functional Magnetic Resonance Imaging; Functional Laterality; Task Performance; Reading; Fluency 


\section{LANGUAGE ABILITY IN ADULTS}

\section{Introduction}

Language ability variations that occur across a population of healthy adult subjects are to date not sufficiently explored. In the present study we aimed to explore how brain activation related to language ability levels is expressed in adults, without imposing restraints regarding age or pre-defined regions of interest. We have correlated language ability performance scores with brain activation as measured with functional Magnetic Resonance Imaging (fMRI). We investigated reading comprehension with a sentence completion task (SENCO) and word fluency with a word generation task (WORGE).

Reading and reading comprehension brings forth activity in Wernicke's area in the left temporal lobe, denoted as the posterior part of Brodmann area (BA) 22. Commonly, this activation extends to parts of the inferior parietal lobe in BA 39 and 40. Broca's area, located in the left inferior frontal gyrus (IFG) and specified as BA 44 and 45, is often co-activated during reading paradigms (Lindenberg \& Scheef, 2007; Vigneau et al., 2006; Bookheimer, 2002; Friederici, 2002). Word fluency is typically characterized in the brain by activation of Broca's area (Heim, Eickhoff, \& Amunts, 2009), often accompanied by activation in adjacent prefrontal regions and also activation in the temporal and parietal cortices (Friedman et al., 1998; Fu et al., 2002; Gauthier, Duyme, Zanca, \& Capron, 2009). Further, the anterior cingulate cortex (ACC) and the anterior insular cortex (AIC) are frequently engaged across a wide distribution of cognitive tasks, including language (Fu et al., 2002; Medford and Critchley, 2010). Performance differences on language production or perception tasks may be attributed to in- or decreased activation of these areas. However, the subdivision in many linguistic and non-linguistic functions of Broca's area (e.g. Vigneau et al., 2006) and the great variance in definition of Wernicke's area both argue against an approach focusing only on classical left-hemispheric language areas (Bookheimer, 2002; Martin, 2003).

Brain activation related to individual performance on language tasks has been addressed only in a few studies. Booth and colleagues (2003) showed that high performance on a visual spelling task is associated to high activation intensity in the IFG (left BA 46 and right BA 45), whereas high performance on a rhyming task corresponds to increased activation in bilateral IFG (BA 9), bilateral superior temporal gyrus (BA 22) and the left supramarginal gyrus (BA 40). Weber and colleagues (2006) found that performance on a semantic decision task in 


\section{LANGUAGE ABILITY IN ADULTS}

epilepsy patients correlates positively with activation in the left temporoparietal cortex. However, according to the same study, performance correlates negatively with activation in the left inferior frontal cortex. A study adapting a semantic discrimination task showed that children with good reading skills express increased activation in the left BA 22 (Meyler et al., 2007). In another study on children and adults, high word generation performance is predominantly characterized by activation in the right frontal cortex and in medial cortical areas (Brown et al., 2005).

These diverging results cannot readily be unified by a theory on functional support of language-related areas towards better language perception and production. Instead, individual performance may be represented by a general intelligence model. The neural characteristics of intelligence and reasoning are suggested to be distributed according to a structural model linking frontal and parietal regions (Jung \& Haier, 2007). This model is based on a review of functional and structural neuroimaging as well as human lesion studies and overlaps to a large extent language-related areas (Broca's and Wernicke's area, supramarginal and angular gyri). The review suggests that there is a mainly left-lateralized network, involving frontoparietal regions, activated by tasks tapping intelligence and reasoning. The functional correlates of this model may be explained by the neural efficiency hypothesis of intelligence, which states that less activation in involved neural networks is correlated with increased cognitive performance (Haier et al., 1988; Neubauer \& Fink, 2009; Brown et al., 2005; Meyler et al., 2007).

In a previous attempt to characterize the correspondence of individual performance on a wide range of language ability tasks to brain activation, we performed a laterality study investigating differences between left- and right-hemispheric regions of interest (ROIs) (Van Ettinger-Veenstra et al., 2010). We calculated the laterality indices (LI) in ROIs of fourteen of the subjects that were included in the present study while they performed the SENCO task. The LI-scores were correlated with performance results on off-line language ability tests consisting of a reading task (READ), a battery of cognitive performance tasks measuring abstract high-level linguistic abilities (BeSS), a verbal fluency task (FAS), and a picture naming task (BNT). We found that performance on READ correlates with activation in the right-hemispheric posterior temporal lobe and performance on BeSS correlates with activation in the right IFG. Furthermore, higher performance on READ, BeSS, and FAS is associated to less right-ear dominance (indicating less left-hemispheric language dominance 


\section{LANGUAGE ABILITY IN ADULTS}

in the brain) on a dichotic listening paradigm. This increased bilaterality of activation corresponding to better performance may either indicate a higher neural efficiency of the left hemisphere, or advocate an active role for the right hemisphere in representing individual cognitive linguistic abilities.

Our present study aims to identify specific regions in both the left and right hemisphere that are important for normal language ability. For this aim we used sentence completion and word fluency fMRI paradigms to investigate if language ability (based on BeSS, FAS, READ and BNT performance scores) shows to be correlated with brain activation, and to discern which are the candidate areas for language ability modulation processes in the healthy adult brain. 


\section{LANGUAGE ABILITY IN ADULTS}

\section{Results}

\section{1 fMRI paradigm activation}

The SENCO paradigm was associated with a large, highly significant cluster of activation in the left IFG, including Broca's area, and extending to the temporal pole. Also, the middle frontal gyrus was highly active. The left superior (STG) and middle temporal gyrus (MTG) showed large clusters of activation, this included activation in Wernicke's area.

Corresponding areas in the right hemisphere were activated, although with lower amplitude and spread. Furthermore, extensive activation was observed in the visual cortex (extrastriate cortex), extending to the bilateral fusiform area, the ACC, the striatum, and the thalamus.

The WORGE paradigm elicited activation primarily in Broca's area. The activation extended to the left medial frontal gyrus (MFG) and AIC. Left-hemispheric activation was also observed in the fusiform gyrus, the striatum and the network related to eye movements; namely, the frontal eye fields, supplementary eye fields, and the intraparietal sulcus. In the right hemisphere, a cluster of activation was observed in the AIC, extending to the putamen. In addition, the ACC was significantly activated.

\subsection{Language ability}

We applied multiple regression analysis to investigate the contribution of performance scores to brain activation (see Table I for results). Off-line READ and BeSS performance scores correlated with neural activity resulting from the SENCO paradigm predominantly in regions in the right hemisphere (Fig 1). In the frontal lobe, increased READ performance was associated with increased activation in the right MFG (BA 9), the right IFG (BA 45, 46, and BA 47), and the right MTG (BA 21). In addition, READ performance was significantly correlated with activation in a small cluster in the left MTG (BA 21/22). No negative correlations with brain activation were observed for READ. Subjects with high BeSS performance elicited increased activation in the right IFG (BA 47), the right MTG (BA 21), and the left hippocampus. There was an overlap with the BA 47 and BA 21 regions that were associated with higher performance on READ. Negative correlations with BeSS performance scores were found in the left hemisphere in frontal lobe areas (BA 9, BA 10), in ACC (BA 32), and in the postcentral gyrus (BA 3). Additionally, BeSS scores were negatively correlated with activation in the right-hemispheric ACC (BA 24) and the superior frontal gyrus (SFG) (BA 8). 


\section{LANGUAGE ABILITY IN ADULTS}

Table I. Identified clusters with significant correlations between fMRI parametric estimates and performance scores on FAS, BNT, READ and BeSS tests. Location labels and Brodmann area numbers are given according to the WFU PickAtlas version 2.4 (Maldjian, Laurienti, Kraft, \& Burdette, 2003). The location of activation is given in MNI coordinates.

$B A=$ Brodmann area, $L=$ left,$R=$ right , Size =cluster size in voxels, $M F G=$ middle frontal gyrus, $I F G=$ inferior frontal gyrus, $S F G=$ superior frontal gyrus, $A C C=$ anterior cingulate cortex, $M T G=$ middle temporal gyrus. The rows in boldface are the locations with significance on the post-hoc ROI analysis, $p<0.05$, ** is $p<0.01$, *** is $p<0.001$.

\begin{tabular}{|c|c|c|c|c|c|c|c|}
\hline \multirow[t]{2}{*}{ Task } & \multirow[t]{2}{*}{ BA } & \multirow[t]{2}{*}{ Location } & \multirow[t]{2}{*}{ Size } & \multirow[t]{2}{*}{ Z } & \multicolumn{3}{|c|}{ MNI coordinates } \\
\hline & & & & & $\mathrm{x}$ & $\mathrm{y}$ & $\mathrm{z}$ \\
\hline \multicolumn{8}{|c|}{ WORGE positive correlations } \\
\hline$F A S$ & 6 & L MFG (posterior) $* * *$ & 6 & 3.82 & -30 & 2 & 56 \\
\hline \multirow[t]{3}{*}{$B N T$} & - & R Subgyral & 5 & 3.60 & 42 & -8 & -22 \\
\hline & $9 / 45$ & R IFG *** & 5 & 3.49 & 54 & 14 & 28 \\
\hline & - & R Uncus & 5 & 3.48 & 18 & -2 & -28 \\
\hline \multicolumn{8}{|c|}{ WORGE negative correlations } \\
\hline \multirow[t]{6}{*}{ BNT } & $10 / 32$ & R SFG (medial) $* * *$ & 12 & 3.71 & 18 & 50 & 6 \\
\hline & 25 & L Caudate & 8 & 3.62 & -10 & 12 & -12 \\
\hline & 45 & L IFG triangularis & 12 & 3.56 & -58 & 22 & 20 \\
\hline & $45 / 46$ & L IFG triangularis ** & 14 & 3.50 & -42 & 32 & 6 \\
\hline & 32 & $\mathbf{R} \mathbf{A C C} * *$ & 15 & 3.37 & 6 & 42 & 16 \\
\hline & 10 & L SFG *** & 9 & 3.31 & -22 & 54 & 4 \\
\hline \multicolumn{8}{|c|}{ SENCO positive correlations } \\
\hline \multirow[t]{12}{*}{ Read } & 46 & R IFG triangularis *** & 67 & 4.40 & 54 & 30 & 12 \\
\hline & 21 & R MTG & 21 & 4.29 & 50 & -12 & -18 \\
\hline & $10 / 47$ & $\begin{array}{l}\text { R IFG triangularis / } \\
\text { orbitalis ** }\end{array}$ & 21 & 3.99 & 40 & 36 & $\mathbf{0}$ \\
\hline & 21 & R MTG (posterior) & 45 & 3.84 & 54 & -52 & 4 \\
\hline & 46 & $\begin{array}{l}\text { R IFG triangularis } \\
\text { (medial) } * *\end{array}$ & 17 & 3.71 & 38 & 28 & 24 \\
\hline & 9 & R MFG *** & 31 & 3.69 & 38 & 36 & 36 \\
\hline & $21 / 22$ & L MTG ** & 8 & 3.66 & -48 & -24 & -2 \\
\hline & 36 & R Fusiform Gyrus *** & 9 & 3.60 & 34 & -32 & -26 \\
\hline & 21 & R MTG (medial) ** & 9 & 3.49 & 56 & -32 & -8 \\
\hline & & $\mathrm{R}$ Anterior cerebellum & 16 & 3.39 & 20 & -46 & -22 \\
\hline & $10 / 47$ & L IFG orbital & 7 & 3.28 & -50 & 48 & -4 \\
\hline & 19 & L Superior occipital & 5 & 3.22 & -24 & -76 & 34 \\
\hline \multirow[t]{3}{*}{ BeSS } & 47 & R IFG & 36 & 3.74 & 42 & 38 & -2 \\
\hline & - & L Hippocampus $* * *$ & 15 & 3.59 & -20 & -34 & 30 \\
\hline & 21 & R MTG *** & 5 & 3.56 & 54 & -54 & 6 \\
\hline \multicolumn{8}{|c|}{ SENCO negative correlations } \\
\hline \multirow[t]{2}{*}{ Read } & 10 & L SFG & 21 & 3.88 & -20 & 56 & 30 \\
\hline & 2 & R Postcentral gyrus & 10 & 3.61 & 36 & -22 & 34 \\
\hline \multirow[t]{7}{*}{ BeSS } & 3 & L Postcentral gyrus & 14 & 4.01 & -52 & -14 & 38 \\
\hline & $46 / 10$ & L MFG *** & 18 & 3.96 & -30 & 44 & 8 \\
\hline & 32 & $\mathbf{L} \mathbf{A C C} * * *$ & 18 & 3.84 & -6 & 48 & 6 \\
\hline & 24 & $\mathbf{R} \mathbf{A C C} * * *$ & 6 & 3.82 & 2 & 24 & -6 \\
\hline & 8 & R SFG *** & 17 & 3.78 & 22 & 34 & 48 \\
\hline & 10 & L SFG *** & 15 & 3.67 & -22 & 58 & 28 \\
\hline & 9/32 & L MFG/SFG $* * *$ & 10 & 3.58 & -4 & 38 & 32 \\
\hline
\end{tabular}




\section{LANGUAGE ABILITY IN ADULTS}

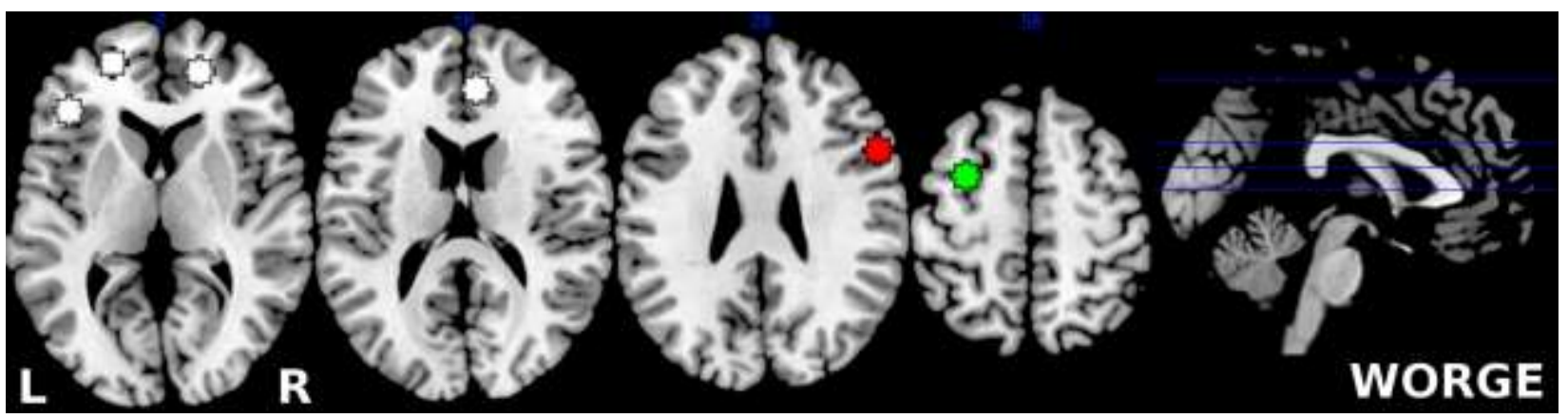

Figure 1. Regions of interest resulting from a multivariate analysis performed on data gathered during the SENCO paradigm. Green (light gray): positive correlation with READ. Red (dark gray): positive correlation with BeSS. White: negative correlation with BeSS. Only regions with a significant correlation of the extracted parametric estimate with the performance scores are shown (in boldface in Table I). The graphs represent the significant correlation of scoring percentages (x-axis) with parametric estimates (y-axis) for the clusters in the right IFG respectively in the right MTG while the regression lines are added to visualize the direction of correlation.

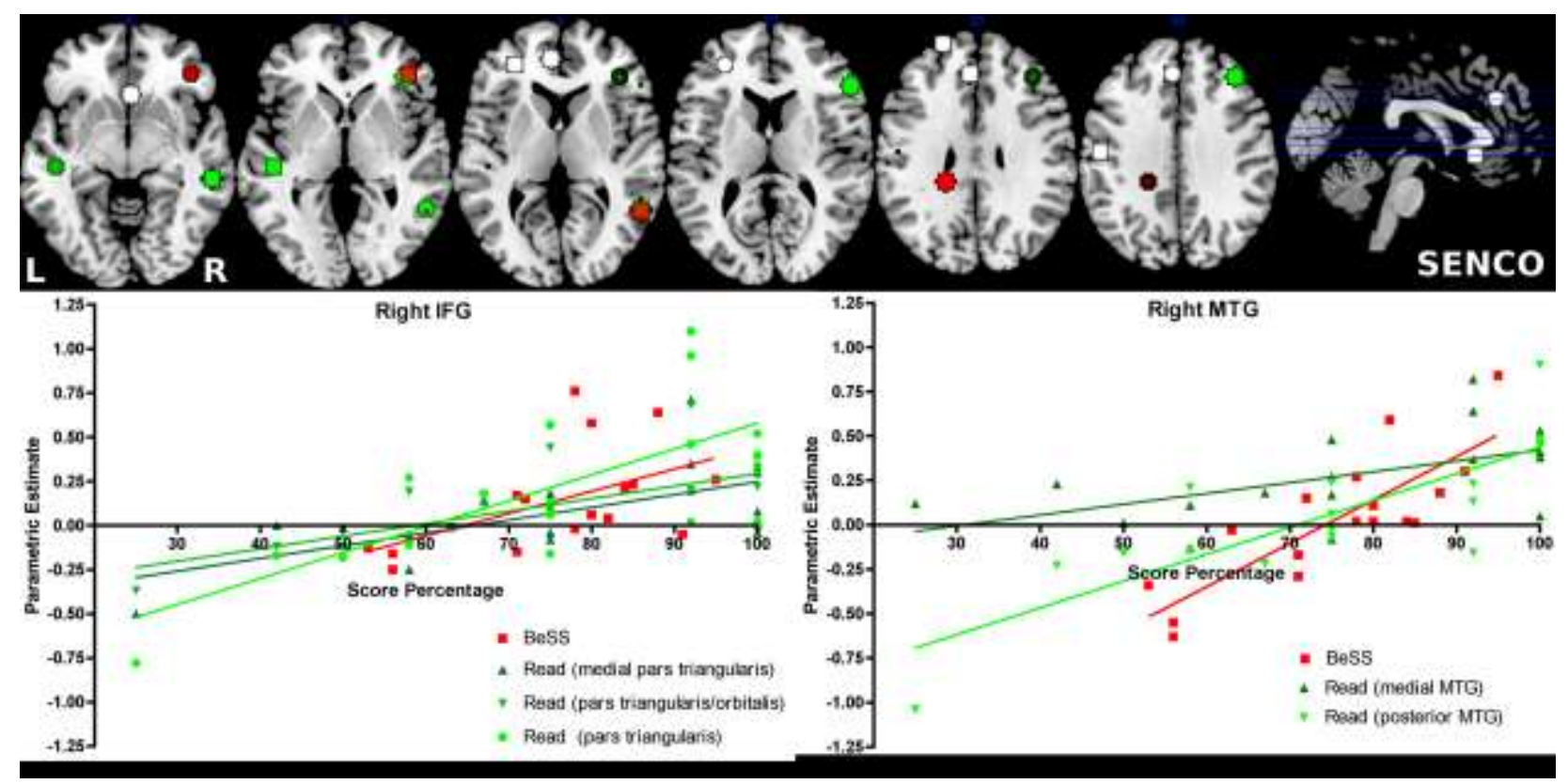

Figure 2. Regions of interest resulting from a multivariate analysis performed on data gathered during the WORGE paradigm. Green (light gray): positive correlation with FAS. Red (dark gray): positive correlation with BNT. White: negative correlation with BNT. Only regions with a significant correlation of the extracted parametric estimate with the performance scores are shown (in boldface in Table I).

Performance scores on the off-line tests FAS and BNT correlated with the on-line test of verbal fluency (WORGE) revealed significant activation in frontal cortical areas (Fig 2). Increased performance on FAS was associated with increased activation in the posterior part of the left MFG. No negative correlations were associated with FAS. Subjects that performed 


\section{LANGUAGE ABILITY IN ADULTS}

high on BNT showed increased right-hemispheric activation in the IFG (BA 9, BA 45). On the other hand, subjects with low performance on BNT showed increased activation in the left SFG (BA 10) and the left IFG (BA 45, BA 46). Additionally, BNT performance scores correlated negatively with activation in the right ACC (BA 32) and the right SFG (BA 10). The activated cluster in the right SFG was located medial to the SFG cluster found in the left hemisphere.

We tested for multicollinearity but found no significant correlations between age and performance scores. We however observed a trend for age * READ (Pearson $r=-0.45, p=$ 0.06 ) which ensued from one outlier, removal resulted in $r=-0.26, p=0.32$. Performance scores on BeSS (mean 150.8, range 106-189 out of 210) and READ (mean 8.9, range 3-12 out of 12) was highly correlated with each other (Pearson $r=0.80, p<0.001$ ). Performance scores on FAS (mean 45, range 27-64) and BNT (mean 56.3, range 50-59 out of 60) did not correlate. 


\section{LANGUAGE ABILITY IN ADULTS}

\section{Discussion}

The present study aimed to investigate neural correlates to language ability by using language performance scores as covariates in a second-level multiple regression analysis of the sentence completion SENCO paradigm and the word generation WORGE paradigm. We did a preliminary analysis of the SENCO and WORGE paradigms with a t-test to determine the elicited activation for all subjects and found results in line with previous studies (Raichle et al., 1994; Bookheimer, 2002; Friederici, 2002; Lindenberg \& Scheef, 2007).

Increased language ability was assessed as a positive correlation between off-line performance scores and brain activation. The SENCO paradigm required semantic understanding of the participants in order for them to generate a word that finished the sentence. The whole timespan, including both the reading and the sentence completion moment, was used in the analysis. Therefore we have correlated SENCO with sentence comprehension performance (READ). BeSS, however, is a more suitable clinical test to measure language ability in subjects with minor language problems since it is less demanding than the high-school-level READ test. (Laakso, Brunnegård, Hartelius, \& Ahlsén, 2000). We included the BeSS test primarily to investigate linguistic ability on a broad spectrum of pragmatic, semantic, and syntactic ability; including repetition, recreating, inference, and comprehension of grammar, words and metaphors. Since BeSS correlates significantly with READ performance results, a specific reading ability test may not be needed in later (patient) studies in order to measure language ability. READ and BeSS performance correlated with activation in the right IFG (BA 47) and MTG (BA 21). Tasks evoking explicitly righthemispheric IFG activation require integration of semantic knowledge and context interpretation (Caplan \& Dapretto, 2001) and also mediation of retrieval processes (Fletcher, Shallice, Frith, Frackowiak, \& Dolan, 1998). Right-sided activation in BA 47 is often linked to decision-making and advanced suppression processes of behavior in tasks related to working memory (Aron, Robbins, \& Poldrack, 2004; Rogers et al., 1999; Völlm et al., 2006), understanding of semantic context (Kang, Constable, Gore, \& Avrutin, 1999) and verb generation (McCarthy, Blamire, Rothman, Gruetter, \& Shulman, 1993). Higher performance in READ and BeSS was also associated to increased activation in the right BA 21, but not in the STG contrasting previous findings (Friederici, Makuuchi \& Bahlmann, 2009). In the left hemisphere, language performance, assessed by READ, correlated with activation in Wernicke's area. However, for the WORGE paradigm, language performance assessed by 


\section{LANGUAGE ABILITY IN ADULTS}

BNT, correlated negatively with activation in Broca's area, corresponding to previous results (Weber et al., 2006).

BNT is often used together with FAS to test fluency on the semantic and phonetic level in patients with aphasia (Rogalski, Rademaker \& Weintraub, 2007). Moreover, both BNT and FAS performance scores correlate with intelligence (Tallberg \& Bergen, 2010). We investigated the nature of the correlations of BNT and FAS with WORGE and have observed clear differences between activation related to FAS performance and activation related to BNT performance. Thus, we cannot argue for a similar nature of naming ability and word fluency ability. Nevertheless, we observed a trend towards a ceiling effect in the BNT scores, likely due to the fact that our test population of adults had a cognitive and linguistic ability within normal range. The difference in activation correlating with high FAS performance and high BeSS/READ performance may indicate the existence of specific functional areas aiding specific linguistic skills rather than a general language ability trait.

For both SENCO and WORGE paradigms, a consistent pattern of increased activation in the lateral prefrontal cortex was observed, in and around the dorsolateral prefrontal cortex (DLPFC: defined as BA 9 and 46). In general, we observed that performance was positively correlated with activity in the right DLPFC and negatively correlated with activity in the left DLPFC. The DLPFC is described by Faw as a coordinating functional area; "dominant in directing attention, working memory, and willed action" (2003, p. 85) and its main function is thought to be response selection (Badre \& Wagner, 2004). Activation in the left DLPFC has been observed previously during a verbal fluency paradigm (Ravnkilde, Videbech, Rosenberg, Gjedde, \& Gade, 2002). A dissociation between the left and the right DLPFC has been observed previously related to attention during the conflict-evoking STROOP task. Activity in the left DLPFC was observed to be related to pre-conflict expectations towards the preparation of higher cognitive processes whereas the right DLPFC showed a postconflict role, related to monitoring and controlling the execution of a task (Vanderhasselt, De Raedt, \& Baeken, 2009). Another study showed a dissociation in mid-DPLFC related to planning; left DLPFC activation related to high goal hierarchy demands (which steps to execute to obtain a goal) and right DLPFC activation related to high search depth demands ('mentally looking ahead') (Kaller, Rahm, Spreer, Weiller \& Unterrainer, 2011). Our finding of a dissociation may reflect a left DLPFC role for executing a step-wise task (such as reading and completing a sentence) on a basic level, while the right DLPFC aids in finding 


\section{LANGUAGE ABILITY IN ADULTS}

more economical solutions reviewing the problem as a whole. Therefore, activation in the right DLPFC might indicate a more profound insight in - or better monitoring of - the presented task, attributing to a greater language proficiency (conform Kang et al., 1999; Caplan \& Dapretto, 2001; Bookheimer, 2002).

We have chosen to analyze the results of the multivariate regression post-hoc using a correlation of parametric estimates with performance scores since no correction for multiple measurements could be applied, likely caused by the relatively small group size (Thirion et al., 2007). These correlations add no significance to the results but rather illustrate how individual performance scores are related to the strength of activation in each subject. Since we have found clusters of activation in overlapping areas based on different multivariate regressions, we believe that our results with its restrictions may serve subsequent studies on language abilities.

\subsection{Conclusions}

Results in this study showed that performance scores on off-line language ability tasks correlated with brain activity predominantly in the right hemisphere (BA 47 and BA 21). That is to say, results in this whole-brain, subregion-specific study are in line with previous findings based on laterality indices in gross regions (Van Ettinger-Veenstra et al., 2010). Our findings of more right-hemispheric activation in the IFG, DLPFC, and the temporal lobe for higher language ability clearly indicate that cognitively demanding linguistic tasks are positively modulated in the right hemisphere. In contrast, the left DLPFC shows activation related to normal or reduced language ability. 


\section{LANGUAGE ABILITY IN ADULTS}

\section{Materials and Methods}

Eighteen healthy subjects (females/males $=9 / 9$; mean age $=40 \mathrm{y}$, range $=21-64$ y) were recruited. The subjects were right-handed according to the Edinburgh handedness inventory (Oldfield, 1971), had no contraindication for MR safety and reported no history of neurological, cognitive or psychiatric disorders, including alcohol and drug abuse or pathological language problems. Examination of the MRI images did not reveal any individual brain anomalies. The subjects signed written informed consents and the study was approved by the regional Ethics Committee of Linköping. The test procedure lasted a total of two to three hours, distributed over two separate days. On the first occasion, the subjects attended a meeting to obtain information on the study, and they completed a reading comprehension test. On the second occasion the remaining language ability tests were administered, immediately followed by the fMRI examination.

\subsection{Language Ability Tests}

The language tests administered off-line were designed to examine language ability in healthy subjects. A Swedish translation of the Test of Language Competence (TLC) was used to investigate higher cognitive language abilities by investigating applied semantics, syntax and pragmatics (Wiig \& Secord, 1985) (see van Ettinger-Veenstra et al., 2010 for details). Word retrieval was tested with the Boston Naming Test (BNT) (Tallberg, 2005) and a verbal fluency test (FAS) (Friedman et al., 1998; Loonstra, Tarlow, \& Sellers, 2001). Language comprehension was measured with the BeSS test that determines subtle speech and language disorders (Laakso et al., 2000) and a reading comprehension task (READ) selected from the Swedish Scholastic Assessment Test (SeSAT) which is an instrument for selection to higher education (Stage \& Ögren, 2004).

\section{2 fMRI Examination}

FMRI acquisition parameters and the sentence completion paradigm (SENCO) measuring reading comprehension are described in (Van Ettinger-Veenstra et al., 2010). For this study, we did not differentiate between the sentence reading and sentence completion moment but analyzed the activation related to 8 subsequent incomplete sentences and the pause in which the sentence was completed as one block. The word generation paradigm (WORGE) where the subjects were instructed to silently generate words beginning with a given letter is described in (Engström et al., 2010), with a control condition presented similarly but existing 


\section{LANGUAGE ABILITY IN ADULTS}

of a star, alternating with a row of stars.

\subsection{Data Analysis}

FMRI SPM5 (http://www.fil.ion.ucl.ac.uk/spm/software/spm5/) was used for the analysis of the fMRI data. Functional scans were motion corrected, resliced, normalized at $2 \times 2 \times 2 \mathrm{~mm}^{3}$ to a standard brain (Montreal Neurological Institute or MNI space), and smoothed with an $8 \mathrm{~mm}$ FWHM Gaussian kernel to allow for group analysis. For one subject, nr 18, it was necessary to include movement parameters in the analysis to minimize movement artifacts. Two other subjects moved significantly during one out of two fMRI sessions (movement $>3 \mathrm{~mm}$ ). They were therefore removed from the respective analyses.

A general linear model (GLM) was used to retrieve functional information and analyze individual results contrasting task and control blocks. The resulting contrast files were tested against zero with a one-sample t-test applying false discovery rate correction (Benjamini \& Hochberg, 1995) with $\mathrm{p}=0.01$ as significance threshold.

\subsection{1 fMRI versus Language Ability}

We analyzed the brain activation related to performance with second-level multiple regression analyses, incorporating performance scores translated into z-scores as covariate. To control for the age differences, age was included as a nuisance variable. This resulted in four analyses; two to examine the relation between WORGE and FAS, and WORGE and BNT and two to examine the relation between SENCO and READ, and SENCO and BeSS. The resulting statistical maps were thresholded at $\mathrm{p}<0.001$ (uncorrected) with an extent threshold of 5 voxels (see results in Table 1).

\subsubsection{Post-hoc Parametric Estimate Analysis}

The second-level statistical maps were masked with an inclusive anatomical mask of the brain, excluding midbrain structures. Around each observed cluster a spherical ROI with a radius of $6 \mathrm{~mm}\left(\sim 1 \mathrm{ml}\right.$ or $\left.984 \mathrm{~mm}^{3}\right)$ was built in the MarsBaR toolbox (Brett, Anton, Valabregue, \& Poline, 2002). Clusters directly adjacent to or located partly in ventricles and inferior orbitofrontal cortex where EPI (echo planar imaging) artifacts and movement artifacts were highly likely to occur were discarded. The ROIs were applied to individual, unsmoothed images and parametric estimates were extracted from each ROI. These parametric estimates, or effect sizes, were correlated with individual performance scores. 


\section{LANGUAGE ABILITY IN ADULTS}

Furthermore, the ROIs were applied to the statistical map of the group analysis and parametric estimates were extracted and tested for their significance. The ROIs whose effect sizes taken from the group analysis statistical map were significant and moreover correlated significantly ( $\mathrm{p}<0.05$ or less where denoted) with performance in language ability tests are reported in boldface in Table I. The figures were made with MRIcron (Rorden, Karnath \& Bonilha, 2007) and GraphPad Prism software. 


\section{LANGUAGE ABILITY IN ADULTS}

\section{Acknowledgments}

The authors thank Gunnel Förhammar, Jennie Pettersson and Cecilia Nellie for support during the design and administration of the language ability tests. Ståhls Foundation and the Strategic Research Area of the Center of Medical Image Science and Visualization (CMIV) at Linköping University, Sweden are acknowledged for financial support. 


\section{LANGUAGE ABILITY IN ADULTS}

\section{References}

Aron, A. R., Robbins, T. W., \& Poldrack, R. A. (2004). Inhibition and the right inferior frontal cortex. Trends Cogn. Sci., 8(4), 170-177.

Badre, D., \& Wagner, A. D. (2004). Selection, integration, and conflict monitoring; assessing the nature and generality of prefrontal cognitive control mechanisms. Neuron, 41(3), 473-487.

Benjamini, Y., \& Hochberg, Y. (1995). Controlling the False Discovery Rate: A Practical and Powerful Approach to Multiple Testing. J. R. Statist. Soc. B, 57(1), 289-300.

Bookheimer, S. (2002). Functional MRI of language: new approaches to understanding the cortical organization of semantic processing. Annu. Rev. Neurosci., 25, 151-188.

Booth, J. R., Burman, D. D., Meyer, J. R., Gitelman, D. R., Parrish, T. B., \& Mesulam, M. M. (2003). Relation between brain activation and lexical performance. Hum. Brain Mapp., 19(3), 155-169.

Brett, M., Anton, J. L., Valabregue, R., \& Poline, J. B. (2002). Region of interest analysis using an SPM toolbox[abstract] Presented at the 8th International Conference on Functional Mapping of the Human Brain, June 2-6, Sendai, Japan. Available on CDROM in NeuroImage, 16(2).

Brown, T. T., Lugar, H. M., Coalson, R. S., Miezin, F. M., Petersen, S. E., \& Schlaggar, B. L. (2005). Developmental changes in human cerebral functional organization for word generation. Cereb. Cortex, (New York, N.Y.: 1991), 15(3), 275-290.

Caplan, R., \& Dapretto, M. (2001). Making sense during conversation: an fMRI study. Neuroreport, 12(16), 3625-3632.

Engström, M., Karlsson, M., Croné, M., Ragnehed, M., Antepohl, W., Landtblom, A.-M., \& Lundberg, P. (2010). Clinical fMRI of language function in aphasic patients: reading paradigm successful, while word generation paradigm fails. Acta Radiol., 51(6), 679- 


\section{LANGUAGE ABILITY IN ADULTS}

686.

Faw, B. (2003). Pre-frontal executive committee for perception, working memory, attention, long-term memory, motor control, and thinking: A tutorial review. Conscious. Cogn., 12(1), 83-139.

Fletcher, P. C., Shallice, T., Frith, C. D., Frackowiak, R. S., \& Dolan, R. J. (1998). The functional roles of prefrontal cortex in episodic memory. II. Retrieval. Brain, $121(\mathrm{Pt}$ 7), 1249-1256.

Friederici, A. (2002). Towards a neural basis of auditory sentence processing. Trends Cogn. Sci., 6(2), 78-84.

Friederici, A., Makuuchi, M., \& Bahlmann, J. (2009). The role of the posterior superior temporal cortex in sentence comprehension. Neuroreport, 20(6), 563-568.

Friedman, L., Kenny, J. T., Wise, A. L., Wu, D., Stuve, T. A., Miller, D. A., Jesberger, J. A., et al. (1998). Brain activation during silent word generation evaluated with functional MRI. Brain Lang., 64(2), 231-256.

Fu, C. H., Morgan, K., Suckling, J., Williams, S. C., Andrew, C., Vythelingum, G. N., \& McGuire, P. K. (2002). A functional magnetic resonance imaging study of overt letter verbal fluency using a clustered acquisition sequence: greater anterior cingulate activation with increased task demand. NeuroImage, 17(2), 871-879.

Gauthier, C. T., Duyme, M., Zanca, M., \& Capron, C. (2009). Sex and performance level effects on brain activation during a verbal fluency task: a functional magnetic resonance imaging study. Cortex, 45(2), 164-176.

Haier, R., Siegel, B., Nuechterlein, K., Hazlett, E., Wu, J., Paek, J., Browning, H., et al. (1988). Cortical glucose metabolic rate correlates of abstract reasoning and attention studied with positron emission tomography. Intelligence, 12(2), 199-217.

Heim, S., Eickhoff, S. B., \& Amunts, K. (2009). Different roles of cytoarchitectonic BA 44 


\section{LANGUAGE ABILITY IN ADULTS}

and BA 45 in phonological and semantic verbal fluency as revealed by dynamic causal modelling. NeuroImage, 48(3), 616-624.

Jung, R. E., Haier, R., J. (2007). The parieto-frontal integration theory (P-FIT) of intelligence: converging neuroimaging evidence. Behav. Brain Sci. 30(2), 135-154.

Kaller, C. P., Rahm, B., Spreer, J., Weiller, C., Unterrainer, J. M., (2011). Dissociable contributions of left and right dorsolateral prefrontal cortex in planning. Cereb. Cortex, 21(2), 307-317.

Kang, A. M., Constable, R. T., Gore, J. C., \& Avrutin, S. (1999). An event-related fMRI study of implicit phrase-level syntactic and semantic processing. NeuroImage, 10(5), 555561.

Laakso, K., Brunnegård, K., Hartelius, L., \& Ahlsén, E. (2000). Assessing high-level language in individuals with multiple sclerosis: a pilot study. Clin. Linguist. Phon., 14(5), 329.

Lindenberg, R., \& Scheef, L. (2007). Supramodal language comprehension: role of the left temporal lobe for listening and reading. Neuropsychologia, 45(10), 2407-2415.

Loonstra, A. S., Tarlow, A. R., \& Sellers, A. H. (2001). COWAT metanorms across age, education, and gender. Appl. Neuropsychol., 8(3), 161-166.

Martin, R. C. (2003). Language processing: Functional organization and neuroanatomical basis. Annu. Rev. Psychol., 54, 55-89.

McCarthy, G., Blamire, A. M., Rothman, D. L., Gruetter, R., \& Shulman, R. G. (1993). Echoplanar magnetic resonance imaging studies of frontal cortex activation during word generation in humans. Proc. Natl. Acad. Sci. U.S.A., 90(11), 4952-4956.

Meyler, A., Keller, T. A., Cherkassky, V. L., Lee, D., Hoeft, F., Whitfield-Gabrieli, S., Gabrieli, J. D., et al. (2007). Brain activation during sentence comprehension among good and poor readers. Cereb. Cortex, 17(12), 2780-2787. 


\section{LANGUAGE ABILITY IN ADULTS}

Medford, N., \& Critchley, H. D. (2010). Conjoint activity of anterior insular and anterior cingulate cortex: awareness and response. Brain Struct. Funct., 214(5-6), 535-549.

Neubauer, A. C., \& Fink, A. (2009). Intelligence and neural efficiency. Neurosci. Biobehav. Rev., 33(7), 1004-1023.

Oldfield, R. C. (1971). The assessment and analysis of handedness: the Edinburgh inventory. Neuropsychologia, 9(1), 97-113.

Raichle, M. E., Fiez, J. A., Videen, T. O., MacLeod, A. K., Pardo, J. V., Fox, P. T., \& Peterse, S. E. (1994). Practice-related changes in human brain functional anatomy during nonmotor learning. Cereb. Cortex, 4, 8-26.

Ravnkilde, B., Videbech, P., Rosenberg, R., Gjedde, A., \& Gade, A. (2002). Putative tests of frontal lobe function: A PET-study of brain activation during Stroop's test and verbal fluency. J. Clin. Exp. Neuropsychol., 24, 534-547.

Rogalski, E., Rademaker, A., Weintraub, S. (2007). Primary progressive aphasia: Relationship between gender and severity of language impairment. Cog. Behav. Neurol., 20, 38-43.

Rogers, R. D., Owen, A. M., Middleton, H. C., Williams, E. J., Pickard, J. D., Sahakian, B. J., \& Robbins, T. W. (1999). Choosing between small, likely rewards and large, unlikely rewards activates inferior and orbital prefrontal cortex. J. Neurosci., 19(20), 90299038.

Rorden, C., Karnath, H. O., Bonilha, L. (2007). Improving lesion-symptom mapping. J. Cogn. Neurosci. 19(7), 1081-1088.

Stage, C., \& Ögren, G. (2004). The Swedish Scholastic Assessment Test (SweSAT). Development, Results and Experiences ( No. (EM No. 49)). Umeå: Umeå University, Department of Educational Measurement. Retrieved from http://www8.umu.se/edmeas/hprov/english/hp-publ_eng.html

Tallberg, I. M. (2005). The Boston Naming Test in Swedish: normative data. Brain Lang., 


\section{LANGUAGE ABILITY IN ADULTS}

94(1), 19-31.

Tallberg, I. M. \& Bergendal, G. (2010). Strategies of lexical substitution and retrieval in multiple sclerosis. Aphasiology, 23(9), 1184-1195.

Thirion, B., Pinel, P., Tucholka, A., Roche, A., Ciuciu, P., Mangin, J.-F., \& Poline, J.-B. (2007). Structural analysis of fMRI data revisited: improving the sensitivity and reliability of fMRI group studies. IEEE Trans. Med. Imaging, 26(9), 1256-1269.

Van Ettinger-Veenstra, H. M., Ragnehed, M., Hällgren, M., Karlsson, T., Landtblom, A.-M., Lundberg, P., \& Engström, M. (2010). Right-hemispheric brain activation correlates to language performance. NeuroImage, 49(4), 3481-3488.

Vanderhasselt, M.-A., De Raedt, R., \& Baeken, C. (2009). Dorsolateral prefrontal cortex and Stroop performance: Tackling the lateralization. Psychon. Bull. Rev., 16(3), 609-612.

Vigneau, M., Beaucousin, V., Hervé, P. Y., Duffau, H., Crivello, F, Houdé, O., Mazoyer, B., et al. (2006). Meta-analyzing left hemisphere language areas: phonology, semantics, and sentence processing. NeuroImage, 30(4), 1414-1432.

Vigneau, M., Beaucousin, V., Hervé, P.-Y., Jobard, G., Petit, L., Crivello, Fabrice, Mellet, E., et al. (2011). What is right-hemisphere contribution to phonological, lexico-semantic, and sentence processing? Insights from a meta-analysis. NeuroImage, 54(1), 577-593.

Völlm, B., Richardson, P., McKie, S., Elliott, R., Deakin, J. F., \& Anderson, I. M. (2006). Serotonergic modulation of neuronal responses to behavioural inhibition and reinforcing stimuli: an fMRI study in healthy volunteers. Eur. J. Neurosci., 23(2), $552-560$.

Weber, B., Wellmer, J., Schur, S., Dinkelacker, V., Ruhlmann, J., Mormann, F., Axmacher, N., et al. (2006). Presurgical language fMRI in patients with drug-resistant epilepsy: effects of task performance. Epilepsia, 47(5), 880-886.

Wiig, E., \& Secord, W. (1985). Test of Language Competence. Toronto, Canada: Merill. 